\title{
NEW EMERGING FIELDS OF APPLICATION OF PROPOLIS
}

\author{
Vassya Bankova*, Milena Popova, Boryana Trusheva \\ Institute of Organic Chemistry with Centre of Phytochemistry, \\ Bulgarian Academy of Sciences, 1113 Sofia, Bulgaria \\ *bankova@orgchm.bas.bg
}

\begin{abstract}
Propolis (bee glue) is a sticky resinous material applied by honey bees Apis mellifera L. as a building material in their hives and as a defensive substance against infections. Propolis has been used as a remedy in traditional medicine systems all over the world, mainly to treat wounds and burns, sore throat, stomach ulcer, etc. Modern science has confirmed the antimicrobial and antiviral action of propolis, and has discovered numerous other beneficial pharmacological properties of bee glue: immunomodulating, anti-inflammatory, antiobesity, antitumor, and many others. For this reason, a significant number of products containing propolis have been developed and commercialized: medical devices, over-the-counter preparations, health foods and beverages, cosmetics. This review does not deal with propolis applications in the improvement and protection of human health. Instead, it is focused on some new and promising areas of propolis use and innovative propolis-containing products that have emerged in the last few years: improving the growth performance of livestock, food preservation, food packaging, textile materials for biomedical application, etc.
\end{abstract}

Keywords: propolis; livestock growth performance; food preservatives; food packaging

\section{НОВИ ПОЛИЬА ВО ЗАРОДИШ ЗА ПРИМЕНА НА ПРОПОЛИС}

Прополисот е леплив смолест материјал кој медоносните пчели, Apis mellifera L., го користат како градежен материјал за нивните улишта и како одбранбена супстанција од инфекции. Прополисот се користи и како лек во традиционалната медицина ширум светот, главно за лекување на рани и изгореници, болки во грлото, чир на желудникот итн. Модерната наука го потврди антимикробното и антивирусно дејство на прополисот и откри бројни други негови корисни фармаколошки својства како што се: имуномодулаторно, антиинфламаторно, против прекумерна телесна тежина, антитуморно и уште многу други. Поради сето ова се развиени и комерцијализирани голем број производи кои содржат прополис: медицински средства, препарати кои се продаваат без лекарски рецепт, здрава храна и напивки, козметички производи. Овој преглед не се занимава со употребата на прополисот за подобрување и заштита на човековото здравје. Наместо тоа, фокусот е на некои нови и ветувачки области за негова примена и на иновативни производи кои содржат прополис и кои се појавија во последните години, имено: подобрувања во одгледувањето на добиток, конзервирање на храна, пакување на храна, текстилни материјали за биомедицинска примена итн.

Клучни зборови: прополис; одгледување на добиток; конзерванси за храна; пакување на храна

\section{INTRODUCTION}

Propolis (bee glue) is a sticky resinous material applied by honey bees Apis mellifera L. as a building material in their hives. Bees use it to block holes and cracks and to cover the internal walls of the hive. Propolis is also an important element of the so-called social immunity of honey 
bees: it can be considered a part of the bees' social immune system, providing the colony as a whole with some general defence against infections and parasites [1] because of its antimicrobial properties.

Bees produce propolis from lipophylic resinous materials which they collect from different plant parts (buds, young leaves, resins, etc.) by mixing them with wax and saliva [2]. They take the material for propolis production from plant excretions, which protect vulnerable parts of living plants from microbial infections. Thus, honeybees make use of the biosynthetic capacity of plants and utilize the secondary plant metabolites, forming the resins, for the same purpose as the plants: for protection. As a result, the activity against diverse microorganisms is an inherent property of propolis, a fact recognized by human beings millennia ago. Propolis has been used as a remedy in traditional medicine systems all over the world, mainly to treat wounds and burns, sore throat, stomach ulcer, etc. [3]. Modern science has confirmed the antimicrobial and antiviral action of propolis, and has discovered numerous other beneficial pharmacological properties of bee glue: immunomodulating, anti-inflammatory, antiobesity, antitumor, and many others [4]. For this reason, a significant number of products containing propolis have been developed and commercialized: medical devices, over-the-counter preparations, health foods and beverages, cosmetics. The interest of consumers and the demand for such products is steadily growing [5].

It is very important to note that the chemical composition of propolis is far from constant. It varies significantly depending on the specific plant resin used by the honeybees in propolis production. At different locations with their particular climatic and phyto-geographic conditions, the plant sources are different and as a result the chemistry of propolis alters dramatically [6]. This could be a grave problem in propolis standardization and quality control [6], although serious efforts have been made to overcome this problem and some results have already been achieved [7]. Notably, although of different chemical composition, propolis from different locations always demonstrates considerable biological activity $[8,9]$.

Obviously, propolis has a number of applications in the improvement and protection of human health. However, in this review we will not deal with these applications. Instead, we will focus on some new and promising areas of propolis use and innovative propolis-containing products that have emerged in the last few years.

\section{PROPOLIS CHEMISTRY AND CHEMICAL DIVERSITY}

Whatever the suggested applications of propolis, its use is based on its chemical and biological properties, and these properties are determined by the chemical constituents of the propolis. As already mentioned, propolis chemistry is highly variable; it depends on the source plant and respectively on the specificity of the local flora at the site of collection, as well as on the preference of honeybees towards specific available botanical sources. A brief overview of published chemical data reveals that in a particular phyto-geographic region, bees demonstrate a constant and unambiguous preference for particular propolis source plants, and for this reason, different propolis types exist (for recent review articles on propolis chemistry, see [10-13]).

It is important to note that different propolis types contain constituents of diverse structural types, mainly phenolics of different classes. Basic information on the most widespread propolis chemical types and their major constituents is presented in Table 1.

The useful properties of propolis are due to its plant-derived constituents but, surprisingly, the different chemical composition does not always lead to significant differences in pharmacological and physicochemical properties. Nevertheless, some specificity has been observed; for example, the diterpene-rich Mediterranean propolis has weak antioxidant activity, in contrast to the polyphenol-rich types (especially Brazilian green and poplar type), while its antibacterial properties are comparable to those of poplar type. For this reason, studies of any type of propolis application must always be accompanied by chemical characterization of the particular propolis used in the experiments, or at least by determining the chemical type of this propolis. During the last decade, the awareness that propolis is a product of variable chemical composition and that there are many propolis types became common knowledge. Nevertheless, a significant number of studies on propolis pharmacology and applications are yet published without any chemical analysis of the bee glue, and this makes them practically useless. This is most unfortunate because, among them, there are investigations of high quality, regarding the biological and technological content, and also ones that report valuable applications. 
Table 1

Characteristic constituents of most widespread chemical types of propolis

\begin{tabular}{|c|c|c|c|c|}
\hline Geographical origin & Propolis type & $\begin{array}{l}\text { Basic source } \\
\text { plant source }\end{array}$ & Characteristic constituents & References \\
\hline $\begin{array}{l}\text { Europe, Western Asia, } \\
\text { China, South Africa, } \\
\text { North America, } \\
\text { New Zealand }\end{array}$ & Poplar type & $\begin{array}{l}\text { Bud exudates of } \\
\text { polar trees, mainly } \\
\text { Populus nigra }\end{array}$ & $\begin{array}{l}\text { Pinocembrin, galangin, chrysin, } \\
\text { pinobanksin 3-O-acetate, pentenyl } \\
\text { caffeates, phenethyl caffeate (CAPE), } \\
\text { caffeic acid, ferulic and isoferulic acid }\end{array}$ & [14-20] \\
\hline $\begin{array}{l}\text { Brazil (Sao Paulo, } \\
\text { Rio de Janeiro, } \\
\text { Minas Gerais) }\end{array}$ & Brazilian green & $\begin{array}{l}\text { Leaf exudates of } \\
\text { Baccharis } \\
\text { dracunculifolia } \\
\end{array}$ & $\begin{array}{l}\text { Artepillin } \mathrm{C} \text {, baccharin, druparin, } \\
\text { aromadendrine, caffeoylquinic acids }\end{array}$ & {$[21,22]$} \\
\hline $\begin{array}{l}\text { Brazil (North-East part), } \\
\text { Cuba, Mexico }\end{array}$ & South American red & $\begin{array}{l}\text { Exudates of } \\
\text { Dalbergia } \\
\text { ecastophyllum, other } \\
\text { Dalbergia species }\end{array}$ & $\begin{array}{l}\text { Medicarpin, vestitol, } \\
\text { 3-O-methyl vestitol }\end{array}$ & [23-25] \\
\hline $\begin{array}{l}\text { Southern Greece and } \\
\text { Greek islands, Crete, } \\
\text { Sicily, Malta, } \\
\text { Adriatic Coast of } \\
\text { Croatia }\end{array}$ & Mediterranean & $\begin{array}{l}\text { Cupressus } \\
\text { sempervirens resin }\end{array}$ & $\begin{array}{l}\text { Diterpenic acids (cupressic, } \\
\text { isocupressic, imbricataloic), } \\
\text { totarol, ferruginol }\end{array}$ & [26-29] \\
\hline $\begin{array}{l}\text { Indoinesia, Oman, } \\
\text { Brazil, Myanmar, } \\
\text { Thailand }\end{array}$ & Mangifera type & $\begin{array}{l}\text { Mangifera indica } \\
\text { fruit exudate }\end{array}$ & $\begin{array}{l}\text { Cardols, cardanols, anacardic acids, } \\
\text { cycloartane triterpenes }\end{array}$ & {$[30-34]$} \\
\hline
\end{tabular}

\section{NEW FIELDS OF PROPOLIS APPLICATION}

The information concerning uses of propolis in areas other than human medicine and quality of life is represented in Table 2, grouped in accordance with the specific applications.

One of these areas is the use of propolis in improving the growth performance and productivity of livestock. The test subjects have been different animal species, including poultry (chickens, laying hens, quails) [35-48], lambs [49, 50], cattle [51], sheep [52], pigs [53], and fish: sea bass [54], rainbow trout [55-57], Nile tilapia [58-60], and carp [61] (Table 2). In general, propolis has a positive effect on the biochemical parameters, growth and productivity of the test animals, and is regarded as a possible alternative to antibiotics in animal feed. In addition, it has the advantage of not inducing resistance in microorganisms [120, 121].

It is important to note that in the literature there are many articles dealing with the effect of propolis on the growth and productivity of domestic animals, many more than the references cited in Table 2. The reason for not mentioning them in this review is the fact that their authors have not presented any information on the propolis used: neither the geographic origin, nor chemical data. In many cases, there are no data even about the method of extraction or treatment of the propolis in order to administer it to the test animals. Such re- sults are irreproducible and have no real scientific value, thus they have not been summarized here.

Another area of intensive research in the last few years is the application of propolis in the preservation of foods. Food preservatives include mainly antimicrobials and antioxidative agents. Antimicrobials added to foods serve two purposes: to control natural spoilage of food and to avoid/control contamination by microorganisms, including pathogenic ones (of food safety concern) [122]. On the other hand, food antioxidants are used for extending shelf life and impeding decay [123], mainly by preventing lipid peroxidation and rancidification, which are the most common types of oxidation occurring in foodstuffs during storage [124]. Propolis favorably brings together antioxidant and antimicrobial properties, and low toxicity. This remarkable combination makes it an excellent candidate for preserving different foods. Successful experiments have been performed for utilization of propolis extracts in the preservation of numerous products: fruit juices [62-65], fruits [68-77], vegetables [78-85], eggs [86-91], meat and fish products [92-100] (Table 2). This field of application of propolis is rapidly growing, as indicated by the growing number of publications. In addition, these studies as a rule include a reasonable chemical characterization of the propolis used. However, the large-scale commercial use of propolis as a food preservative is not yet a reality, because it would require proper standardization. 
T a ble 2

New emerging propolis applications

\begin{tabular}{|c|c|c|c|}
\hline Propolis applications & Propolis extract & Propolis type / origin & Reference \\
\hline 1 & 2 & 3 & 4 \\
\hline \multicolumn{4}{|c|}{ Growth performance improvement of lifestock } \\
\hline \multicolumn{4}{|c|}{ 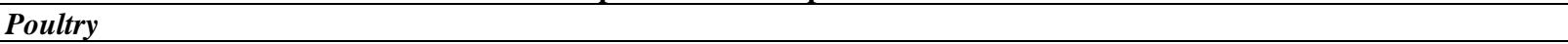 } \\
\hline Ross Broiler Chickens & 96\% Ethanol extract & Iran & [35] \\
\hline Ross 308 Broiler Chick (heat stressed) & $70 \%$ Ethanol extract & Eastern Anatolia & {$[36,37]$} \\
\hline Broiler chicks (male Ross 708) & - & Brazilian green type & [38] \\
\hline Broiler chickens & $70 \%$ Ethanol extract & Poplar type, Iran & [39] \\
\hline Broilers (Ross 308) & $70 \%$ Ethanol extract & Poplar type, China & [40] \\
\hline Broiler chickens & $50 \%$ Ethanol extract & $\begin{array}{l}\text { Adquirió en Maringá-PR, } \\
\text { Brazil }\end{array}$ & [41] \\
\hline Chickens Hubbard JV & $80 \%$ Ethanol extract & Slovak Republic & [42] \\
\hline Chicken hybrid combination Ross 308 & $80 \%$ Ethanol extract & Slovak Republic & [43] \\
\hline Meat-type chicken Nanbu Kashiwa & $\begin{array}{l}\text { Propolis residue after } \\
\text { alcohol extraction }\end{array}$ & Iwate, Japan & [44] \\
\hline Broiler chick & Alcoholic extract & Brazilian green type & [45] \\
\hline Local quails & Raw propolis & Duhok, Iraq & [46] \\
\hline Laying hens, heat stressed & $70 \%$ Ethanol extract & Eastern Anatolia & [47] \\
\hline Hens' egg incubation & - & $\begin{array}{l}\text { Central zone of Republic of } \\
\text { Moldova }\end{array}$ & [48] \\
\hline \multicolumn{4}{|l|}{ Ungulate domestic animals } \\
\hline Lambs & $70 \%$ Ethanol extract & Brazilian green propolis & [49] \\
\hline Lambs (Santa Inês x Dorper) & $70 \%$ Ethanol extract & Brazilian red propolis & [50] \\
\hline Crossbred ( $1 / 2$ Angus $v s .1 / 2$ Nellore) bulls & Ethanol extract & Maringá PR, Brazil & [51] \\
\hline Castrated male sheep & $70 \%$ Ethanol extract & Pernambuco State, Brazil. & [52] \\
\hline Growing pigs & $70 \%$ Ethanol extract & Poplar propolis & [53] \\
\hline \multicolumn{4}{|l|}{ Fish } \\
\hline $\begin{array}{l}\text { Juvenile European sea bass (Dicentrarchus } \\
\text { labrax L.) at low temperature stress }\end{array}$ & Water extract & $\begin{array}{l}\text { Dalmatia region, } \\
\text { middle Croatia }\end{array}$ & [54] \\
\hline Rainbow trout (Oncorhynchus mykiss) & 95\% Ethanol extract & Kunming, China & [55] \\
\hline Rainbow trout (Oncorhynchus mykiss) & 96\% Ethanol extract & Iran & [56] \\
\hline Rainbow trout (Oncorhynchus mykiss) & $80 \%$ Ethanol extract & Urmia, Iran & [57] \\
\hline Nile tilapia (Oreochromis niloticus) & Water extract & Egypt & [58] \\
\hline Nile tilapia (Oreochromis niloticus) & Cereal alcohol extract & Brazilian brown propolis & [59] \\
\hline Nile tilapia (Oreochromis niloticus) fingerlings & Ethanol extract & Dakahlia province, Egypt & {$[60]$} \\
\hline Carp (Cyprinus carpio) & $70 \%$ Ethanol extract & Poplar type & [61] \\
\hline \multicolumn{4}{|c|}{ Preservative effect in food industry } \\
\hline \multicolumn{4}{|l|}{ Beverages } \\
\hline $\begin{array}{l}\text { Unpasteurized fruit juices (apple, orange, } \\
\text { white grape and mandarin) }\end{array}$ & Ethanol extracts & Turkey & [62] \\
\hline Unpasteurized juices & $30 \%$ Alcohol extract & $\begin{array}{l}\text { Kozie Kąty apriary (southern } \\
\text { Poland). }\end{array}$ & [63] \\
\hline Apple juice & $80 \%$ Ethanol & Kayseri, Turkey. & [64] \\
\hline Apple juice & $\begin{array}{l}\text { Food supplement containing } \\
\text { raw propolis }\end{array}$ & Poplar type propolis, Spain & [65] \\
\hline Production of distilled beverages & Ethanol extracts & $\begin{array}{l}\text { Bebedouro-SP, Brazil: } \\
\text { green propolis; } \\
\text { Formiga-MG, Brazil: } \\
\text { brown propolis }\end{array}$ & [66] \\
\hline Distilled beverage (sugarcane spirit) & $80 \%$ Ethanol extract & $\begin{array}{l}\text { Brazilian green and brown } \\
\text { propolis type }\end{array}$ & [67] \\
\hline \multicolumn{4}{|l|}{ Fruits } \\
\hline Mandarins & Ethyl acetate extract & Hebei Province, China & [68] \\
\hline Orange & 96\% Ethanol extract & Iraq (Baghdad) & [69] \\
\hline Apple & $96 \%$ Ethanol extract & Iraq (Baghdad) & [70] \\
\hline Sweet cerry & Ethanol and water extracts & Hatay province, Turkey & [71] \\
\hline Grapefruit cv. Star Ruby & $70 \%$ Ethanol extract & Hatay province, Turkey & [72] \\
\hline 'Kent' mango fruits & $70 \%$ Ethanol extract & Brazilian green type & [73] \\
\hline Dragon fruit & $80 \%$ Ethanol extract & $\begin{array}{l}\text { Selangor state Malaysia; } \\
\text { China }\end{array}$ & {$[74,75]$} \\
\hline Papaya & 95\% Ethanol extract & China & [76] \\
\hline
\end{tabular}




\begin{tabular}{|c|c|c|c|}
\hline$\overline{1}$ & $\overline{2}$ & 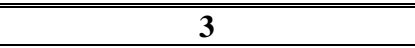 & 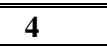 \\
\hline Papaya (Carica papaya L. cv. Hawaiiana) & Ethanol extract & $\begin{array}{l}\text { Llanta Azul apiary, Zaragoza } \\
\text { (Antioquia - Colombia) }\end{array}$ & {$[77]$} \\
\hline \multicolumn{4}{|l|}{ Vegetables } \\
\hline Rice (Oryza sativa, var. hinohikari) & $\begin{array}{l}\text { Absolute ethanol, methylene } \\
\text { chloride and hexane extracts }\end{array}$ & Brazilian green propolis & {$[78]$} \\
\hline Carrot (minimally processed) & $\begin{array}{l}25 \% \text { Water and alcohol } \\
\text { extracts }\end{array}$ & Brazilian green propolis & [79] \\
\hline Lettuce & Ethanol extract & Bragança, Portugal & {$[80]$} \\
\hline Cucumber & 70\% Ethanol extracts & Pato Branco (PR), Brazil & {$[81]$} \\
\hline Beans, carrots against white rot disease & Alcohol extract & Iraq & {$[82]$} \\
\hline Mixed vegetables for soup & Propolis extract & Argentina & [83] \\
\hline $\begin{array}{l}\text { Celery, leek and butternut squash } \\
\text { (minimally processed) }\end{array}$ & $\begin{array}{l}\text { Ethanolic extract; } \\
\text { standardized to } 10 \% \text { propolis } \\
\text { extract }\end{array}$ & Mendoza province, Argentina & {$[84]$} \\
\hline Chilli (Capsicum annuum L.) & Ethanol extract & Brazilian green propolis & {$[85]$} \\
\hline \multicolumn{4}{|l|}{ Eggs } \\
\hline Eggs quality during storage & $70 \%$ Ethanol extract & Hatay province, Turkey & {$[86,87]$} \\
\hline Japanese quail eggs & $70 \%$ Ethanol extract & Hatay province, Turkey & [88-90] \\
\hline Hens eggs & Ethanol extract & Parana state, Brazil, & [91] \\
\hline \multicolumn{4}{|l|}{ Meat products } \\
\hline Fresh oriental sausage & 95\% Ethanol extract & Cairo, Egypt & [92] \\
\hline Sausages & $96 \%$ Ethanol extract & Boyacá, Colombia & [93] \\
\hline Fermented meat sausage "Alheira" & $95 \%$ ethanol & $\begin{array}{l}\text { Vila Franca, Viana do Castelo, } \\
\text { Portugal }\end{array}$ & [94] \\
\hline Beef patties & Commercial ethanol extracts & Sonora, Mexico, poplar type & [95] \\
\hline Poultry meat product-cured cooked ham & $96 \%$ Ethanol extract & Slovakia & [96] \\
\hline \multicolumn{4}{|l|}{ Fish products } \\
\hline $\begin{array}{l}\text { Nile tilapia (Oreochromis niloticus) fillets, } \\
\text { frozen storage }\end{array}$ & Water extract & Egypt & [97] \\
\hline $\begin{array}{l}\text { Cachama fish (Piaractus brachypomus) fillets } \\
\text { (refrigeration) }\end{array}$ & $96 \%$ Ethanol extract & Boyacá, Colombia & [98] \\
\hline Fresh fish burgers & $70 \%$ Ethanol extract & La California, LI, Italy & [99] \\
\hline Fresh shibuta (Barbus grypus) fillets & Water extract & $\begin{array}{l}\text { Fanus Natural Company, } \\
\text { Trabzon, Turkey }\end{array}$ & {$[100]$} \\
\hline \multicolumn{4}{|l|}{ Others } \\
\hline Milk protein concentrate (diary beverages) & $\begin{array}{l}94 \% \text { Ethanol extract } \\
\text { Water extract }\end{array}$ & Canada & {$[101]$} \\
\hline \multicolumn{4}{|c|}{ Food packaging } \\
\hline Chitosan-propolis coated polypropylene films & $80 \%$ Ethanol extract & Konya region, Turkey. & {$[102]$} \\
\hline Antimicrobial edible coating of Bell Pepper & 95\% Ethanol extract & China & [103] \\
\hline $\begin{array}{l}\text { Active film with antimicrobial effect, reduces } \\
\text { oxidation of butter during storage }\end{array}$ & $70 \%$ Ethanol extract & Brazilian red propolis & {$[104]$} \\
\hline $\begin{array}{l}\text { Lactic acid polymer packaging films containing } \\
\text { propolis }\end{array}$ & $\begin{array}{l}\text { Standardized propolis extract } \\
\text { EPID }^{\circledR}\end{array}$ & Italy & {$[105]$} \\
\hline Cassava starch films containing propolis & $\begin{array}{l}\text { Commercial ethanolic propo- } \\
\text { lis extract Brazil, } 12 \%(\mathrm{w} / \mathrm{v}) \\
\text { dry solid }\end{array}$ & Brazil & [106] \\
\hline \multicolumn{4}{|c|}{ Textile materials for biomedical applications } \\
\hline $\begin{array}{l}\text { Antibacterial activity, water repellent and UV } \\
\text { protection of cotton textiles }\end{array}$ & $70 \%$ Ethanol extract & Monofia province, Egypt & {$[107]$} \\
\hline Antibacterial finishing of cotton fabrics & $30 \%$ Ethanol extract & North-East Romania & {$[108]$} \\
\hline $\begin{array}{l}\text { Electrospun propolis/polyurethane composite } \\
\text { nanofibers }\end{array}$ & THF/DMF solution & Australia & [109] \\
\hline $\begin{array}{l}\text { Electrospun bicomponent mats of poly(vinyl } \\
\text { alcohol) (PVA) and aqueous propolis solution }\end{array}$ & Water extracts & Vilnius, Lithuania & [110] \\
\hline $\begin{array}{l}\text { Orally fast dissolving fibers by electrospinning } \\
\text { process with polyvinyl pyrrolidone (PVP) and } \\
\text { polyvinyl alcohol (PVA) }\end{array}$ & $70 \%$ Ethanol extract & $\begin{array}{l}\text { Chiangmai Healthy Product } \\
\text { Co., Ltd. (Thailand) }\end{array}$ & [111] \\
\hline \multicolumn{4}{|c|}{ Other applications } \\
\hline $\begin{array}{l}\text { Inhibition of corrosion for carbon steel } \\
\text { in aqueous media }\end{array}$ & Water propolis extract & $\begin{array}{l}\text { Poplar type } \\
\text { Daqahlia province (delta Nile), } \\
\text { Egypt }\end{array}$ & [112] \\
\hline $\begin{array}{l}\text { Propolis as a novel membrane and its } \\
\text { application in phenol biosensors }\end{array}$ & Acetone extract & $\begin{array}{l}\text { Urmia, } \\
\text { northwestern Iran }\end{array}$ & {$[113]$} \\
\hline
\end{tabular}




\begin{tabular}{lllc}
\hline \hline \multicolumn{1}{c}{ 1 } & \multicolumn{1}{c}{$\mathbf{2}$} & $\mathbf{3}$ & $\mathbf{4}$ \\
\hline $\begin{array}{l}\text { Propolis in organic/inorganic interfaces } \\
\text { optoelectronic applications }\end{array}$ & $96 \%$ Ethanol extract & Ukraine & {$[114,115]$} \\
\hline $\begin{array}{l}\text { Nanodiamond Restorative Materials containing } \\
\text { propolis (hydrogel) }\end{array}$ & Hydroalcoholic extracts & $\begin{array}{l}\text { Australia } \\
\text { Brazilian green propolis type } \\
\text { Uruguyan Propolis }\end{array}$ & {$[116]$} \\
\hline $\begin{array}{l}\text { Application to air filters (in air purifiers or } \\
\text { heating, ventilation, and air-conditioning } \\
\text { (HVAC) systems) }\end{array}$ & Ethanol extract & Korea & {$[117,118]$} \\
\hline $\begin{array}{l}\text { Effect on the ruminal degradation of nutrients } \\
\text { and reduction of methanogenesis in ruminants }\end{array}$ & $70 \%$ ethanol extract & $\begin{array}{l}\text { Brazilian red } \\
\text { Egyptian brown }\end{array}$ & {$[119]$} \\
\hline \hline
\end{tabular}

Closely related to food preservation is the application of propolis as a constituent of the socalled active packaging materials, based on the incorporation of active substances, mainly natural antimicrobial and antioxidant agents, in food biopackaging materials [105]. Its incorporation in polylactic acid films, and films obtained from starch and cellulose nanocrystals, has given excellent results in the preservation of cheese, butter [104] and bell peppers [103]. It has been confirmed that propolis can be used successfully in a coating of gelatin or cellulose nanocrystals onto plastic films, whereas coating of propolis directly on cellulosic materials is not suitable as a packaging material because of the lack of stability of the polyphenol fraction [125]. The diffusivity of propolis compounds in polylactic acid polymer for the development of anti-microbial packaging films has also been studied [105].

Incorporation of propolis in textiles for producing antimicrobial textile materials for different purposes, such as wound dressing, tissue engineering, medical and hygienic use, has also been studied. Propolis has been used either by the incorporation of a small amount of bee glue in electrospun polymer microfibers $[109,110]$, or as an ecofriendly finish for cotton fabric within the scope of a green strategy $[107,108]$.

A few other, unexpected applications of propolis are presented in Table 2 under Others: as a corrosion inhibitor, as a pesticide in horticulture, for optoelectronic applications, in air filters, etc. Propolis could even contribute to the actions against global warming, because it reduces the methanogenesis in ruminants [119]. Only time will tell whether these applications will be widely accepted.

\section{CONCLUSIONS}

The review of recent propolis literature demonstrates the potential of propolis to be used for the development of diverse innovative products, not only medicines and cosmetics but also in the field of the food industries, packaging, animal husbandry, etc. For this to happen, the combined efforts of researchers and technologists from different areas will be needed in order to make better use of bee glue.

\section{REFERENCES}

[1] M. Simone, J. D. Evans, M. Spivak, Resin collection and social immunity in honey bees, Evolution 63, 30163022 (2009). DOI: 0.1111/j.1558-5646.2009.00772.x.

[2] E. Crane, Beekeeping: Science, Practice and World Recourses, Heinemann, London, 1988.

[3] E. L. Ghisalberti, Propolis: A review, Bee World 60, 5984 (1979). DOI: 10.1080/0005772X.1979.11097738.

[4] J. M. Sforcin, V.Bankova, Propolis: is there a potential for the development of new drugs? J. Ethnopharmacol. 133, 253-260 (2011). DOI: 10.1016/j.jep.2010.10.032.

[5] D. Suarez, D. Zayas, F. Guisado, Propolis: Patents and technology trends for health applications, Journal of Business Chemistry 2, 119-125 (2005).

[6] V. Bankova, Chemical diversity of propolis and the problem of standardization, J. Ethnopharmacol. 100, 114-117 (2005). DOI: 10.1016/j.jep.2005.05.004.

[7] M. Popova, V. Bankova, S. Bogdanov, I. Tsvetkova, C. Naydenski, G.- L. Marcazzan, A.-G. Sabatini, Chemical characteristics of poplar type propolis of different geographic origin, Apidologie 38, 306-311 (2007). DOI: 10.1051/apido:2007013.

[8] A. Kujumgiev, I. Tsvetkova, Y. Serkedjieva, V. Bankova, R. Christov, S. Popov, Antibacterial, antifungal and antiviral activity of propolis of different geographic origin, J. Ethnopharmacol. 64, 235-240 (1999)q. DOI: 10.1016/S0378-8741(98)00131-7.

[9] V. Seidel, E. Peyfoon, D.G. Watson, J. Fearnley, Comparative study of the antibacterial activity of propolis from different geographical and climatic zones. Phytother. Res. 22,1256-1263 (2008). DOI: 10.1002/ptr.2480.

[10] P. Ristivojević, J. Trifković, F. Andrić, D. MilojkovićOpsenica, Poplar-type propolis: chemical composition, botanical origin and biological activity, Nat. Prod. Commun. 10, 1869-1876 (2015).

[11] S. Huang, C. P. Zhang, K. Wang, G. Q. Li, F.L. Hu, Recent advances in the chemical composition of propolis, Molecules 19, 19610-19632 (2014). DOI: 10.3390/molecules191219610.

[12] V. Bankova, B. Trusheva, Chemical profiles of different ptopolis types in relation to their biological activity. In: T. Farooqui, A. Farooqui (Eds), Beneficial Effects of Propolis on Human Health and Chronic Diseases. NOVA Science Publishers, New York, 2013, pp. 1-38. 
[13] M. P. Popova, Structural basis of antimicrobial activity of Mediterranean propolis, In: T. Farooqui, A. Farooqui (Eds), Beneficial Effects of Propolis on Human Health, NOVA Science Publishers, New York, 2013, pp. 171-188.

[14] V. S. Bankova, S. S. Popov, N. L. Marekov, A study on flavonoids of propolis, J. Nat. Prod. 46, 471-474 (1983). DOI: $10.1021 / \mathrm{np50028a007.}$

[15] E. Nagy, V. Papay, G. Litkei, Z. Dinya, Investigation of the chemical constituents, particularly the flavonoid components of propolis and Populi gemma by the GC/MS method, L. Farkas, M. Gabor, F. Kallay (Eds), Flavonoids and Bioflavonoids. Amsterdam: Elsevier, 1985, pp. 233-240.

[16] W. Greenaway, T. Scaysbrook, F. R. Whatley, Composition of propolis of Oxfordshire, UK and its relation to poplar bud exudate. Z. Naturforsch. 43c, 301-304 (1988). DOI: 10.1515/znc-1988-3-423.

[17] V. Bankova, A. Dyulgerov, S. Popov, L. Evstatieva, L. Kuleva, O. Pureb, Z. Zamjansan, Propolis produced in Bulgaria and Mongolia: phenolic compounds and plant origin, Apidologie 23, 79-85 (1992). DOI: 10.1051/apido:19920109.

[18] K. R. Markham, K. A. Mitchell, A. L. Wilkins, J. A. Daldy, Y. Lu, HPLC and GC-MS identification of the major organic constituents in New Zealand propolis, Phytochemistry 42, 205-211 (1996).

DOI: $10.1016 / 0031-9422(96) 83286-9$.

[19] A. C. H. F. Sawaya, D. M. Tomazela, I. B. S. Cunha, V. S. Bankova, M. C. Marcucci, A. R. Custodio, M. N. Eberlin, Electrospray ionization mass spectrometry fingerprinting of propolis, Analyst. 129, 739-744 (2004). DOI: $10.1039 / \mathrm{B} 403873 \mathrm{H}$.

[20] W. Cao, J. Fu, Z. Suo, W. Chen, J. Zheng, A comparative study on chemical compositions of Chinese propolis and poplar resin, Food Fermen. Indust. 33, 162-166 (2007).

[21] V. Bankova, G. Boudourova-Krasteva, J. M. Sforcin, X. Frete, A. Kujumgiev, R. Maimoni-Rodella, S. Popov, Phytochemical evidence for the plant origin of Brazilian propolis from Sao Paulo state, Z. Naturforsch. C. 54, 401-405 (1999). DOI: 10.1515/znc-1999-5-616.

[22] E. W. Teixeira, G. Negri, R. M. S. A. Meira, D. Message, A. Salatino, Plant origin of green propolis: bee behavior, plant anatomy and chemistry, eCAM. 2, 85-92 (2005). DOI: 10.1093/ecam/neh055.

[23] A. Daugsch, C. S. Moraes, P. Fort, Y. K. Park, Brazilian red propolis - chemical composition and botanical origin, eCAM. 5, 435-441 (2008). DOI: $10.1093 /$ ecam/nem057.

[24] C. Lotti, M. C. Fernandez, A. L. Piccinelli, O. CuestaRubio, I. M. Hernandez, L. Rastrelli, Chemical constituents of red Mexican propolis, J. Agric. Food Chem. 58, 2209-2213 (2010). DOI: 10.1021/jf100070w.

[25] A. L. Piccinelli, C. Lotti, L. Campone, O. Cuesta-Rubio, M. C. Fernandez, L. Rastrelli, Cuban and Brazilian red propolis: botanical origin and comparative analysis by high-performance liquid chromatography-photodiode array detection/electrospray ionization tandem mass spectrometry, J. Agric. Food Chem. 59, 6484-6491 (2011). DOI: $10.1021 / \mathrm{jf} 201280 \mathrm{z}$.
[26] B. Trusheva, M. Popova, V. Bankova, I. Tsvetkova, C. Naydenski, A. G. Sabatini, A new type of European propolis, containing bioactive labdanes, Rivista Italiana EPPOS. 13, 3-8 (2003).

[27] E. Melliou, I. Chinou, Chemical analysis and antimicrobial activity of Greek propolis, Planta Med. 70, 15 (2004). DOI: 10.1055/s-2004-827150.

[28] M. P. Popova, K. Graikou, I. Chinou, V. S. Bankova, GC-MS profiling of diterpene compounds in Mediterranean propolis from Greece, J. Agric. Food Chem. 58, 3167-3176 (2010). DOI: 10.1021/jf903841k.

[29] M. Popova, B. Trusheva, S. Cutajar, D. Antonova, D. Mifsud, C. Farrugia, V. Bankova, Identification of the plant origin of the botanical biomarkers of Mediterranean type propolis, Nat. Prod. Commun. 7(5), 569570 (2012).

[30] B. Trusheva, M. Popova, E. B. Koendhori, I. Tsvetkova, H. Naydenski, V. Bankova, Indonesian propolis: chemical composition, biological activity and botanical origin, Nat. Prod. Res. 25, 606-613 (2011). DOI: $10.1080 / 14786419.2010 .488235$.

[31] M. Popova, R. Dimitrova, H. T. Al-Lawati, I. Tsvetkova, H. Najdenski, V. Bankova, Omani propolis: chemical profiling, antibacterial activity and new propolis plant sources, Chem. Cent. J. 7, 158 (2013). DOI: $10.1186 / 1752-153 X-7-158$.

[32] M. do S. S. da Silva, A. M. das G. L. Citó, M. H. Chaves, J. A. D. Lopes, Triterpenóides tipo cicloartano de propolis de Teresina - PI, Quim. Nova, 28, 801-804 (2005). DOI: 10.1590/S0100-40422005000500013.

[33] F. Li, S. Awale, Y. Tezuka, S. Kadota, Cytotoxic constituents of propolis from Myanmar and their structure-activity relationship, Biol. Pharm. Bull. 32, 2075-2078 (2009). DOI: 10.1248/bpb.32.2075.

[34] S. Sanpa, M. Popova, T. Tunkasiri, S. Eitssayeam, V. Bankova, P. Chantawannakul, Chemical profiles and antimicrobial activities of Thai propolis collected from Apis mellifera, Chiang Mai J. Sci. (2015) (in press).

[35] S. K. Shalmany, M.Shivazad, The effect of diet propolis supplementation on Ross Broiler chicks performance, Int. J. Poult. Sci. 5, 84-88 (2006). DOI: 10.3923/ijps.2006.84.88.

[36] P. T. Seven, I. Seven, Effect of dietary Turkish propolis as alternative to antibiotic on performance and digestibility in broilers exposed to heat stress. J. Appl. Anim. Res., 34, 193-196 (2008).

DOI: $10.1080 / 09712119.2008 .9706970$.

[37] P. Tatli Seven, I. Seven, M. Yilmaz, U. G. Şimşek, The effects of Turkish propolis on growth and carcass characteristics in broilers under heat stress, Anim. Feed Sci. Technol. 146, 137-148 (2008). DOI: 10.1016/j.anifeedsci.2007.11.003.

[38] U. T. Mahmoud, M. A. Abdel-Rahman, M. H. A. Darwish, T. J. Applegate, H-w. Cheng, Behavioral changes and feathering score in heat stressed broiler chickens fed diets containing different levels of propolis, Appl. Animal Behav. Sci. 166, 98-105 (2015). DOI: 10.1016/j.applanim.2015.03.003.

[39] A. Daneshmand, G. H. Sadeghi, A. Karimi, A. Vaziry, S. A. Ibrahim, Evaluating complementary effects of ethanol extract of propolis with the probiotic on growth 
performance, immune response and serum metabolites in male broiler chickens, Livestock Science 178, 195201 (2015). DOI: 10.1016/j.livsci.2015.04.012.

[40] M. A. M. Mahmoud, H. S. Abdel-Mohsein, M. R. F. Farghali, Antioxidant properties of Chinese propolis in Ross broilers exposed to heat stress in Egypt, Open $J$. Vet. Med. 5, 197-209 (2015). DOI: $10.4236 /$ ojvm. 2015.59027.

[41] C. Eyng, A. E. Murakami, I. C. Ospina-Rojas, R. B. Pedroso, T. G. V. Silveira, D. A. L. Lourenço, Efecto de la inclusión dietética de extracto etanólico de propóleos en la inmunidad de pollos de engorde [Effect of diet inclusion of ethanolic extract of propolis on broiler immunity], Arch. Med. Vet. 47, 185-192 (2015). DOI: $10.4067 / \mathrm{s} 0301-732 \times 2015000200009$.

[42] P. Haščík, J. Garlík, I.O.E. Elimam, V. Kňazovická, M. Kačániová, M. Šimko, M. Mellen, Meat performance of chickens Hubbard JV after application of propolis extract, J. Microb., Biotechn. Food Sci. 3, 118-121 (2013).

[43] P. Haščik, I. O. Elimam, M. Kročko, M. Bobko, M. Kačaniová, J. Garlík, M. Šimko, A. A. Saleh, The influence of propolis as supplement diet on broiler meat growth performance, carcass body weight, chemical composition and lipid oxidation stability, Acta Univ. Agric. et Silvic. Mendel. Brun. 63, 411-418 (2015). DOI: 10.11118/actaun 201563020411.

[44] K. Kita, I. R. Ken, C. Akamine, W. Kawada, Y. Shimura, T. Inamoto, Influence of propolis residue on the bacterial flora in the cecum of Nanbu Kashiwa, Japanese Poultry Sci. 51, 275-280 (2014) DOI: $10.2141 /$ jpsa.0130137.

[45] R. F. Paludo, T. G. Petrolli, O. J. Petrolli, E. L. Fiorentin, D. Pavan, Effect of addition of green propolis in broiler chick diets on metabolizable energy utilization and hemathological parameters, Enciclopédia Biosfera, Cientifico Conhecer - Goiânia, 11, 827-836 (2015).

[46] I. T. Tayeb, B. F. Sulaiman, Effect of propolis supplementation on productive performance in local quail, IJAS 4, 621-627 (2014).

[47] P. T. Seven, The effects of dietary Turkish propolis and vitamin $\mathrm{C}$ on performance, digestibility, egg production and egg quality in laying hens under different environmental temperatures, Asian-Australasian J Anim. Sci. 21, 1164-1170 (2008). DOI: 10.5713/ajas. 2008.70605.

[48] E. Scripnic, N. Eremia, Propolis extract used in incubation technology for hens eggs treatment, Scientific Papers. Series D. Animal Science LVIII, 330-333 (2015).

[49] C. C. B. F. Ítavo, M. G. Morais, C. L. Ramos, L. C. V. Ítavo, T. R. Tomich, J. A. da Silva, Green propolis extract as additive in the diet for lambs in feedlot, Rev. Bras. Zootecn. 40, 1991-1996 (2011). DOI: $10.1590 / \mathrm{S} 1516-35982011000900021$.

[50] F. A. O. Baracho, G. F. da Silva, E. H. S. Rocha, J. M. D. Gomes, L. G. de Oliveira, L. T. A. dos Santos, F. A. de Souza, P. M. G. Beelen, Desempenho de cordeiros alimentados com Própolis Vermelha de Alagoas e monensina sódica [Performance of confined lambs fed with Brazilian Red Propolis and monensin sodium] XXV CONGRESSO BRASILEIRO DE ZOOTECNIA ZOOTEC 2015 Dimensões Tecnológicas e Sociais da Zootecnia Fortaleza - CE, 27 a 29 de maio de 2015.
http://zootec2015.com.br/anais/Producao\%20e\%20Nutri cao\%20de\%20Ruminantes/1555.pdf

[51] M. V. Valero, L. M. Zeoula, L. P. P. de Moura, J. B. G. C. Júnior, B. B. Sestari, I. N. do Prado, Propolis extract in the diet of crossbred ( $1 / 2$ Angus vs. $1 / 2$ Nellore) bulls finished in feedlot: animal performance, feed efficiency and carcass characteristics [Extrato de própolis na dieta de machos mestiços ( $1 / 2$ Angus vs. $1 / 2$ Nelore) não castrados terminados em confinamento: desempenho animal, eficiência alimentar e características de carcaça], Semina: Ciências Agrárias, Londrina 36, 1067-1078 (2015). DOI: 10.5433/1679-0359.2015v36n2p1067.

[52] F. G. B. da Silva, S. M. Yamamoto, E. M. S. da Silva, M. A. Á. Queiroz, L. A.Gordiano, M. A. Formiga, Propolis extract and sodium monensin on ruminal fermentation and hematological parameters in sheep, Acta Scientiarum. Animal Sciences Maringá 37, 273280, (2015). DOI: 10.4025/actascianimsci.v37i3.25725.

[53] J. Li, I. H. Kim, Effects of Saccharomyces cerevisiae cell wall extract and poplar propolis ethanol extract supplementation on growth performance, digestibility, blood profile, fecal microbiota and fecal noxious gas emissions in growing pigs. Anim. Sci. J., 85, 698-705 (2014). DOI: 10.1111/asj.12195.

[54] T. Šegvić-Bubić, J. Boban, L. Grubišić, Ž. Trumbić, M. Radman, M. Perčić, R. Čož-Rakovac, Effects of propolis enriched diet on growth performance and plasma biochemical parameters of juvenile European sea bass ( $D i$ centrarchus labrax L.) under acute low temperature stress, Aquaculture Nutrition 19, 877-885 (2013). DOI: 10.1111/anu.12032.

[55] J. Deng, Q. An, B. Bi, Q. Wang, L. Kong, L. Tao, X. Zhang, Effect of ethanolic extract of propolis on growth performance and plasma biochemical parameters of rainbow trout (Oncorhynchus mykiss), Fish Physiol. Biochem. 37, 959-967 (2011). DOI: 10.1007/s10695-011-9493-0.

[56] N. S. Naghavi, F. Zeyghampoor, A. Nazari, E. Shams, Infectivity control of Streptococcus iniae isolated and detected from rainbow trout (Oncorhynchus mykiss) in Iran, by beehive propolis ethanol extract, Indian Journal of Fundamental and Applied Life Sciences 4, 563-569 (2014).

[57] A. Tukmechi, F. Karimi Rad, F. Farrokhi, N. Agh, R. Jalili, The effects of short- and long-term diet supplementation with Iranian propolis on the growth and immunity in rainbow trout (Oncorhynchus mykiss)", IJVR 15, 250-255 (2014).

[58] A. A. Abbass, A. M. El-Asely, M. M. M. Kandiel, Effects of dietary propolis and pollen on growth performance, fecundity and some hematological parameters of Oreochromis niloticus. Turk. J. Fish. Aquat. Sci. 12, 851-859 (2012). DOI: 10.4194/1303-2712-v12_4_13.

[59] F. Meurer, M. M. da Costa, D. A. D. de Barros, S. T. de Oliveira, P. S. da Paixao, Brown propolis extract in feed as a growth promoter of Nile tilapia (Oreochromis niloticus, Linnaeus 1758) fingerlings, Aquaculture Res. 40, 603-608 (2009). DOI: 10.1111/j.1365-2109. 2008.02139.x.

[60] E. Wafaa, I. Doaa, A. El-Murr, M. Rania, Effects of dietary inclusion of black cumin seeds, green tea and propolis extraction growth parameters, body composition and economic efficiency of Nile tilapia, Oreo- 
chromis niloticus, World J. Fish Mar. Sci. 6, 447-452 (2014). DOI: 10.5829/idosi.wjfms.2014.06.05.8632.

[61] M. E. Yonar, S. M. Yonar, A. Pala, S. Silici, N. S. Glam, Trichlorfon-induced haematological and biochemical changes in Cyprinus carpio: ameliorative effect of propolis, Dis. Aquat. Organ. 114, 209-216 (2015). DOI: $10.3354 /$ dao02866.

[62] S. Silici, N. A. Koç, F. Mutlu Sariguzel, O. Sagdic, Mould inhibition in different fruit juices by propolis, $A r$ chiv für Lebensmittelhygiene, 56, 87-90 (2005).

[63] M. Makarewicz, I. Drozdz, T. Tuszynski, The effect of some bee products on microbiological stability of fresh fruit juices, J. Health Sci. 2, 125-140 (2012).

[64] S. Silici, K. Karaman, Inhibitory effect of propolis on patulin poduction of Penicillium expansum in apple juice. J. Food Process. Pres. 38, 1129-1134 (2014). DOI: 10.1111/jfpp.12072.

[65] A. Luis-Villaroya, L. Espina, D. García-Gonzalo, S Bayarri, C. Pérez, R. Pagán, Bioactive properties of a propolis-based dietary supplement and its use in combination with mild heat for apple juice preservation, Int. J. Food Microbiol. 205, 90-97 (2015). DOI: 10.1016/j.ijfoodmicro.2015.03.020.

[66] N. A. Montijo, A. F. Silva, G. H. G. Costa, O. E. Ferreira, M. J. R. Mutton, Yeast CA-11 fermentation in musts treated with brown and green propolis, Afr. J. Microbiol. Res. 8, 3515-3522 (2014). DOI: $10.5897 / A J M R 2014.6937$.

[67] M. J. R. Mutton, J. H. de Oliveira Filho, G. H. G. Costa, J. P. Roviero, L. Aline de Freita, Green and brown propolis: efficient natural biocides for the control of bacterial contamination of alcoholic fermentation of distilled beverage, Food Sci. Technol. (Campinas) 34, 767-772 (2014). DOI: 10.1590/1678-457X.6469.

[68] S. Yang, L. Peng, Y. Cheng, F. Chen, S Pan, Control of citrus green and blue molds by Chinese propolis. Food Sci. Biotechnol. 19, 1303-1308 (2010). DOI: $10.1007 / \mathrm{s} 10068-010-0186-8$.

[69] O. N. Manty, Efficacy evaluation of Iraqi propolis against gray mold of stored orange caused by Penicillium digitatum. Plant Pathology Journal 14, 153-157 (2015). DOI: 10.3923/ppj.2015.153.157.

[70] O. N. Matny, S. H. S. Al-Warshan, A. M. Ali, Antifungal evaluation of Iraqi propolis against Penicillium expansum and mycotoxin production in apple, Int. J. Curr. Microbiol. App. Sci. 4, 399-405 (2015).

[71] E. E. Candir, A. E. Ozdemir, E. M. Soylu, N. Sahinler, A. Gül, Effects of propolis on storage of sweet cherry cultivar Aksehir Napolyon, Asian J. Chem. 21, 2659-2666 (2009).

[72] A. E. Özdemír, E. E. Çandir, M. Kaplankiran, E. M. Soylu, N. Şahínler, A. Gül, The effects of ethanoldissolved propolis on the storage of grapefruit cv. Star Ruby. Turk. J. Agric. For, 34, 155-162 (2010). DOI: $10.3906 /$ tar-0902-50.

[73] B.-H. Mattiuz, M.-N. Ducamp-Collin, C. F. M. Mattiuz, C. Vigneault, K. M. Marques, W. Sagoua, D. Montet, Effect of propolis on postharvest control of anthracnose and quality parameters of 'Kent' mango, Scientia Horticulturae 184, 160-168 (2015). DOI: 10.1016/j.scienta.2014.12.035.
[74] N. Zahid, A. Ali, Y. Siddiqui, M. Maqbool, Efficacy of ethanolic extract of propolis in maintaining postharvest quality of dragon fruit during storage. Posth. Biol. Technol., 79, 69-72 (2013).

DOI:10.1016/j.postharvbio. 2013.01.003.

[75] N. Zahid, A. Ali, P. G.Alderson, M. Maqbool, S. Manickam, Dual mode of action of ethanolic extract of propolis (EEP) for the control of postharvest anthracnose in dragon fruits (Conference Paper). Acta Horticulturae 1012, 711-718 (2013)

[76] A. Ali, C. K. Cheong, N. Zahid, Composite effect of propolis and gum arabic to control postharvest anthracnose and maintain quality of papaya during storage, Int . J. Agric. Biol. 16, 1117-1122, (2014).

[77] E. Barrera, J. Gil, A. Restrepo, K. Mosquera, D. Durango, A coating of chitosan and propolis extract for the postharvest treatment of papaya (Carica papaya $\mathrm{L}$. cv. Hawaiiana) [Un Recubrimiento de quitosano y extracto de propóleos para el manejo poscosecha de la papaya (Carica papaya L. cv. Hawaiiana)], Rev.Fac.Nal.Agr. 68, 7667-7678 (2015). DOI: $10.15446 /$ rfnam.v68n2.50982.

[78] G. A. Griffiths, U. Toshitaka, T. Fumihiko, H. Daisuke, Effect of vapors from fractionated samples of propolis on microbial and oxidation damage of rice during storage, J. Food Eng. 88, 341-352 (2008). DOI: 10.1016/j.jfoodeng.2008.02.019.

[79] O. Kameyama, J. A. Junior, J. M. de Assis Teixeira, N. J. de Andrade, V. P. R. Minin,; L. dos Santos Soares, Propolis extract for sanitation and preservation of minimally processed carrot, Revista Ceres, 55, 218-223 (2008). (in Portuguese)

[80] X. Feás, L. Pacheco, A. Iglesias, L. M. Estevinho, Use of propolis in the sanitization of lettuce. Int. J. Mol. Sci. 15, 12243-12257 (2014). DOI: 10.3390/ijms150712243.

[81] C. A. Guginski-Piva, I. dos Santos, A. Wagner Júnior, D. W. Heck, M. F. Flores, K. Pazolini, Propolis for the control of powdery mildew and the induction of phytoalexins in cucumber [El propóleo para el control de oídio y la inducción de fitoalexinas en pepino], Idesia 33, 3947 (2015). DOI: 10.4067/s0718-34292015000100005.

[82] O. N.Matny, E. K. Abdul-Karim, R. A. Naemah, R. A. Al-Ani, Activity of propolis and Boswellia sp. resins extract against Sclerotinia sclerotiorum causative agent of white rot disease of Phaseolus vulgaris and Daucus carota under storage conditions, J. Exp. Biol. Agric. Sci. 2, 65-71 (2014).

[83] M. V. Alvarez, A. G. Ponce, C. A. Mazzucotelli, M. R. Moreira, The impact of biopreservatives and storage temperature in the quality and safety of minimally processed mixed vegetables for soup, J. Sci. Food Agric. 95, 962-971 (2015). DOI: 10.1002/jsfa.6770.

[84] M. V. Alvarez, G. Ponce, M. R. Moreira, Combined effect of bioactive compounds and storage temperature on sensory quality and safety of minimally processed celery, leek and butternut squash, Journal of Food Safety 35, 560-574 (2015). DOI: 10.1111/jfs.12206.

[85] A. Ali, W. L. Chow, N. Zahid, M. K. Ong, Efficacy of propolis and cinnamon oil coating in controlling postharvest anthracnose and quality of chilli (Capsicum annuum L.) during cold storage, Food Bioprocess. Technol. 7, 2742-2748 (2014). DOI 10.1007/s11947-013-1237-y. 
[86] N. Sahinler, A. Gül, G. Çopur, Chemical composition and preservative effect of Turkish propolis on egg quality during storage, Asian J. Chem. 21, 1877-1886 (2009).

[87] G. Copur, O. Camci, N. Sahinler, A. Gul, The effect of propolis egg shell coatings on interior egg quality, Arch. Geflügelk. 72, 35-40 (2008).

[88] A. Aygun, D. Sert, G. Copur, Effects of propolis on eggshell microbial activity, hatchability, and chick performance in Japanese quail (Coturnix coturnix japonica) eggs, Poult. Sci. 91, 1018-1025 (2012). DOI: $10.3382 / p s .2011-01944$

[89] A. Aygun, D. Sert, Effects of prestorage application of propolis and storage time on eggshell microbial activity, hatchability, and chick performance in Japanese quail (Coturnix coturnix japonica) eggs, Poult. Sci. 92, 3330 3337 (2013). DOI: 10.3382/ps.2013-03291.

[90] G. C. Akpinar, S. Canogullari, M. Baylan, S. Alasahan, A. Aygun, The use of propolis extract for the storage of quail eggs, J. Appl. Poult. Res. 24, 427-435 (2015). DOI: $10.3382 / \mathrm{japr} / \mathrm{pfv} 043$.

[91] J. X. De Carvalho, R. O. Suárez, F. Q. Mendes, R. V. De Barros Fernandes, M. C. Da Cunha, A. M. X. De Carvalho, Increased shelf life of eggs through the use of propolis, Semina: Ciências Agrarias 34, 2287-2296 (2013). (in Portuguese) DOI: $10.5433 / 1679-0359.2013 v 34 n 5 p 2287$.

[92] F. H. Ali, G. M. Kassem, O. A. Atta-Alla, Propolis as a natural decontaminant and antioxidant in fresh oriental sausage, Vet. Ital. 46, 167-172 (2010).

[93] C. Gutiérrez-Cortés, H. Suarez Mahecha, Antimicrobial activity of propolis and its effect on the physicochemical and sensorial characteristics in sausages, Vitae 21, 90 96 (2014).

[94] R. Casquete, S. M. Castro, S. Jácome, P. Teixeira, Antimicrobial activity of ethanolic extract of propolis in "Alheira", a fermented meat sausage, Cogent Food \& Agriculture (2015).

DOI: 10.1080/23311932. 2015.1125774.

[95] R. D. Vargas-Sánchez, G. R. Torrescano-Urrutia, E. Acedo-Félix, E. Carvajal-Millán, A. F. GonzálezCórdova, B. Vallejo-Galland, M. J. Torres-Llanez, A. Sánchez-Escalante, Antioxidant and antimicrobial activity of commercial propolis extract in beef patties, $J$. Food Sci. 79, C1499 - C1504 (2014). DOI: $10.1111 / 1750-3841.12533$

[96] M. Kročko, M. Bobko, O. Bučko, M. Čanigová, V. Ducková, Sensory quality, colour and oxidative stability of cured cooked ham with propolis extract, Potravinárstvo 8, 102-106 (2014). DOI: 10.5219/365.

[97] S. I. A. Hassanin, E.-S. A. El-Daly, Effect of propolis and garlic on Nile Tilapia Oreochromis niloticus fillets during frozen storage, J. Arab. Aquacul. Soc. 8, 237-247 (2013).

[98] H. S. Mahecha, Á. J. Toquica, A. C. D. Moreno, Physicochemical evaluation of Cachama fillets (Piaractus brachypomus) preserved with propolis during storage, Rev. Fac. Nal. Agr. 67, 7229-7236 (2014). DOI: $10.15446 /$ rfnam.v67n1.42653.

[99] S. Spinelli, A. Conte, L. Lecce, A. L. Incoronato, M. A. Del Nobile, Microencapsulated propolis to enhance the antioxidant properties of fresh fish burgers, J. Food Process Eng. 38, 527-535 (2015), DOI: 10.1111/jfpe. 12183.
[100] M. Duman, E. Özpolat, Effects of water extract of propolis on fresh shibuta (Barbus grypus) fillets during chilled storage, Food chem. 189, 80-85 (2015). DOI: 10.1016/j.foodchem.2014.08.091.

[101] S. M. Cottica, H. Sabik, D. Bélanger, H. J. Giroux, J. V. Visentainer, M. Britten, Use of propolis extracts as antioxidant in dairy beverages enriched with conjugated linoleic acid, Eur Food Res Technol 241, 543-551 (2015). DOI: 10.1007/s00217-015-2483-1.

[102] E. Torlak, D. Sert, Effectiveness of chitosan-propolis coated polypropylene films against foodborne pathogens, Int. J. Biol. Macromol. 60, 52-55 (2013). DOI: 10.1016/j.ijbiomac.2013.05.013.

[103] A. Ali, Y. Z. Wei, M. A. Mustafa, Exploiting propolis as an antimicrobial edible coating to control post-harvest anthracnose of Bell Pepper, Packaging Technol. Sci. 28, 173-179 (2015), DOI: 10.1002/pts.2088.

[104] S. S. Costa, J. I. Druzian, B. A. S. Machado, C. O. de Souza, A. G. Guimarães, Bi-functional biobased packing of the cassava starch, glycerol, licuri nanocellulose and red propolis, PLOS ONE 9, e112554 (2014). DOI: 10.1371/journal.pone.0112554.

[105] E. Mascheroni, V. Guillard, F. Nalin, L. Mora, L. Piergiovanni, Diffusivity of propolis compounds in polylactic acid polymer for the development of anti-microbial packaging films, J. Food Eng, 98, 294-301 (2010). DOI: 10.1016/j.jfoodeng.2009.12.028.

[106] G. K. P. de Araújo, S. J. de Souza, M. V. da Silva, F. Yamashita, O. H. Gonçalves, F. V. Leimann, M. A. Shirai, Physical, antimicrobial and antioxidant properties of starch-based film containing ethanolic propolis extract, Int. J. Food Sci. \& Technol. 50, 2080-2087 (2015). DOI: $10.1111 /$ ijfs. 12869 .

[107] S. Sharaf, A. Higazy, A. Hebeish, Propolis induced antibacterial activity and other technical properties of cotton textiles, Int. J. Biol. Macromol. 59, 408-416 (2013). DOI: 10.1016/j.ijbiomac.2013.04.030.

[108] D. Abramiuc, L. Ciobanu, R. Muresan, M. Chiosac, A. Muresan, Antibacterial finishing of cotton fabrics using biologically active natural compounds, Fibers and Polymers 14, 1826-1833 (2013). DOI: $10.1007 / \mathrm{s} 12221-013-1826-4$.

[109] J. I. Kim, H. R. Pant, H.-J. Sim, K. M. Lee, C. S. Kim, Electrospun propolis/polyurethane composite nanofibers for biomedical applications, Mater. Sci. Eng. C 44, 5257 (2014). DOI: 10.1016/j.msec.2014.07.062.

[110] E. Adomavičiūtè, S. Stanys, M. Žilius, V. Briedis, Formation and analysis of electrospun nonwoven mats from bicomponent PVA/aqueous propolis nanomicrofibres, Fibres \& Textile East. Europe 23, 5(113): 35-41 (2015). DOI: 10.5604/12303666.1161754.

[111] C. Asawahame, K. Sutjarittangtham, S. Eitssayeam, Y. Tragoolpua, B. Sirithunyalug, J. Sirithunyalug, Formation of orally fast dissolving fibers containing propolis by electrospinning technique, Chiang Mai Journal of Science 42, 469-480 (2015).

[112] A. S. Fouda, A. Hamdy Badr, Aqueous extract of propolis as corrosion inhibitor for carbon steel in aqueous solutions, African J. Pure Appl. Chem. 7, 350-359 (2013). DOI: 10.5897/AJPAC2013.0524

[113] F. Kheiri, R. E. Sabzi, E. Jannatdoust, H. Sedghi, Acetone extracted propolis as a novel membrane and its ap- 
plication in phenol biosensors: the case of catechol, $J$. Solid State Electrochem. 15, 2593-2599 (2011). DOI: $10.1007 / \mathrm{s} 10008-010-1250-2$.

[114] S. I. Drapak, A. P. Bakhtinov, S. V. Gavrylyuk, Z. D. Kovalyuk, O. S. Lytvyn, The formation of organic (propolis films)/inorganic (layered crystals) interfaces for optoelectronic applications, Superlatt. Microstruc. 44, 563-570 (2008). DOI: 10.1016/j.spmi.2007.12.019.

[115] V. Brus, L. Pidkamin, M. Ilashchuk, P. Maryanchuk, Propolis films for hybrid biomaterial-inorganic electronics and optoelectronics, Applied Optics 53, B121-B127 (2014). DOI: 10.1364/AO.53.00B121.

[116] V. T. Perchyonok, T. Souza, R. Felliti, S. Zhang, S. Grobler, Bio-functional nanodiamond restorative materials containing bio-additives: In vitro approach, Open Journal of Stomatology 5, 117-126 (2015). DOI: $10.4236 /$ ojst.2015.55017.

[117] C. G. Woo, J.-S. Kang, H.-J. Kim, Y.-J. Kim, B. Han, Treatment of air filters using the antimicrobial natural products propolis and grapefruit seed extract for deactivation of bioaerosols, Aerosol Science and Technology 49, 611-619 (2015). DOI: $10.1080 / 02786826.2015 .1054983$.

[118] B. Han, J.-S. Kang, H.-J. Kim, C.-G. Woo, Y.-J. Kim, Investigation of antimicrobial activity of grapefruit seed extract and its application to air filters with comparison to propolis and shiitake, Aerosol and Air Quality Research 15, 1035-1044 (2015). DOI: 10.4209/aaqr. 2014.09.0208.

[119] A. S. Morsy, Y. A. Soltan, S. M. A. Sallam, M. Kreuzer, S. M. Alencar, A. L. Abdalla, Comparison of the in vitro efficiency of supplementary bee propolis extracts of different origin in enhancing the ruminal degradability of organic matter and mitigating the formation of methane, Animal Feed Sci. Technol. 199, 51-60 (2015). DOI: 10.1016/j.anifeedsci.2014.11.004.

[120] A. Salatino, É. W. Teixeira, G. Negri, D. Message, Origin and chemical variation of Brazilian propolis. Evidence-based Complementary and Alternative Medicine.;2, 33-38 (2005). DOI: 10.1093/ecam/neh060.

[121] R. Silva-Carvalho, F. Baltazar, C. Almeida-Aguiar, Propolis: A complex natural product with a plethora of biological activities that can be explored for drug development, Evidence-Based Complementary and Alternative Medicine, 2015, Article ID 206439, 29 pages, (2015). DOI: 10.1155/2015/206439.

[122] M. M. Tajkarimi, S. A. Ibrahim, D. O. Cliver, Antimicrobial herb and spice compounds in food, Food Control 21, 1199-1218 (2010). DOI: 10.1016/j. foodcont.2010.02.003.

[123] B. Nanditha, P. Prabhasankar, Antioxidants in bakery products: a review, Crit. Rev. Food. Sci. Nutr. 49, 1-27 (2009). DOI: 10.1080/10408390701764104.

[124] M. Carocho, M. F. Barreiro, P. Morales, I. C. F. R. Ferreira, Adding molecules to food, pros and cons: A review on synthetic and natural food additives, Comprehensive Reviews in Food Science and Food Safety, 13, 377-399 (2014). DOI: $10.1111 / 1541-4337.12065$.

[125] J. M. Fuentes-Alventosa, S. Farris, F. Li, L. Piergiovanni, E. Mascheroni, New active packaging materials based on propolis, Italian Journal of Food Science, 24, 133-136 (2012). 
